\title{
Thrust Analysis and Experiments on Low-Speed Single-Sided Linear Induction Motor
}

\author{
Jae-Hoon Jeong*, Jang-Young Choi ${ }^{\dagger}$, So-Young Sung**, Jong-Won Park** \\ and Jaewon Lim***
}

\begin{abstract}
When the characteristics of a linear induction motor (LIM) are analyzed using finite element analysis (FEA), it is desirable to set the voltage source as an input. If the voltage source is set as an input in FEA, the leakage inductance and primary resistance of the equivalent circuit must be entered by direct calculation, and the magnetizing inductance and secondary reaction effects are directly considered in FEA. Exact calculation is necessary because the primary winding resistance and leakage inductance directly entered will have a significant effect on the LIM output. Therefore, in this study, we accurately calculated the primary leakage inductance and analyzed the resulting LIM characteristics. We calculated the leakage inductance using an analytical equation and FEA, and we confirmed the accuracy by comparing the results with the value experimentally calculated using a manufactured model. We also analyzed the instrument performance and thrust of the LIM as a function of the difference in the leakage inductance. Finally, we present the conclusions on the precise analysis based on the calculation of the leakage inductance.
\end{abstract}

Keywords: Linear induction motor, LIM, Equivalent circuit parameter

\section{Introduction}

Single-sided linear induction motors (LIMs) are very useful where linear motion is required because they directly produce thrust. Therefore, their application in industry has been increasing [1]. However, the characteristics of LIMs differ from those of conventional rotational induction motors (IMs) owing to their structure. First, the air gap in LIMs is inevitably large to maintain mechanical clearance. Thus, the exiting current is larger, and the power factor and efficiency are lower. Second, because of their mechanical structure, LIMs suffer from a longitudinal end effect resulting from the discontinuity of incoming current and magnetic reluctance. The end effect eventually reduces the output of the device, which is the most significant disadvantage of LIMs [2]. In the present study, finite element analysis (FEA), which enables accurate numeric analysis of geometric structures, was used to interpret the characteristics of LIMs.

When the LIM characteristics are analyzed using FEA, we use either a current or voltage source as an input source. Actual electric machinery is a dependent variable whose voltage is constant, and the electric current changes

$\dagger \quad$ Corresponding Author: Dept. of Electrical Engineering, Chungnam National University, Korea. (choi_jy@cnu.ac.kr)

* Dept. of Electrical Engineering, Chungnam National University, Korea. (tpts@cnu.ac.kr)

** Dept. of Offshore Plant Research Division, Korea Research Institute of Ships and Ocean Engineering, Korea. (riverblu@kriso.re.kr)

*** Dept. of Magnetic Levitation and Linear Drive, Korea Institute of Machinery and Materials, Korea. (einses@kimm.re.kr)

Received: March 5, 2016; Accepted: August 13, 2016 according to the loading conditions. Therefore, when using a constant current source, it is not possible to consider current that varies according to the changing loading conditions. In particular, similar to LIMs, when the circuit constant is structurally unbalanced even after applying a balanced three-phase voltage, calculating the unbalanced current for each phase during operation is impossible [3][4]. In the current study, a voltage generator was used as an input source for FEA. In this work, we therefore accurately calculated the primary leakage inductance and analyzed the resulting LIM characteristics. We calculated the leakage inductance using an analytical equation and FEA and confirmed the accuracy by comparing the results with the value experimentally calculated using a manufactured model. We also analyzed the instrument performance and thrust of an LIM as a function of the difference in the leakage inductance.

\section{Leakage Inductance Calculation of LIMs}

\subsection{Analysis model}

Fig. 1(a) shows the LIM model used to verify the interpretation results, whereas Fig. 1(b) shows the detailed plan of the LIM. It is a six-pole LIM with a speed of 1.6 $\mathrm{m} / \mathrm{s}$. The core material uses PN470, and the secondary conductor is aluminum. Similar to rotary IMs (RIMs), the leakage inductance of LIMs can be investigated by analytical methods using an equivalent circuit model [4]. 

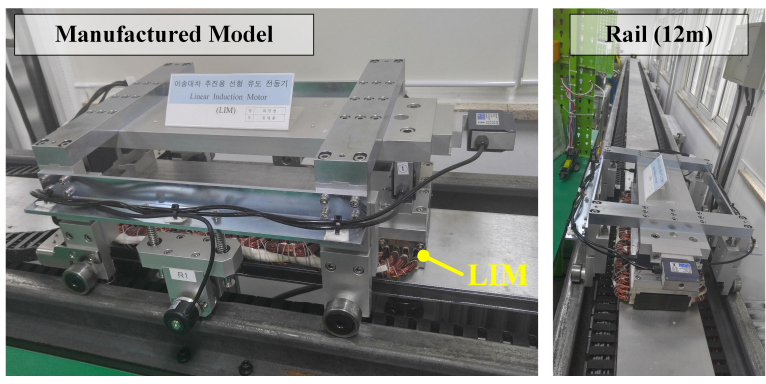

(a)

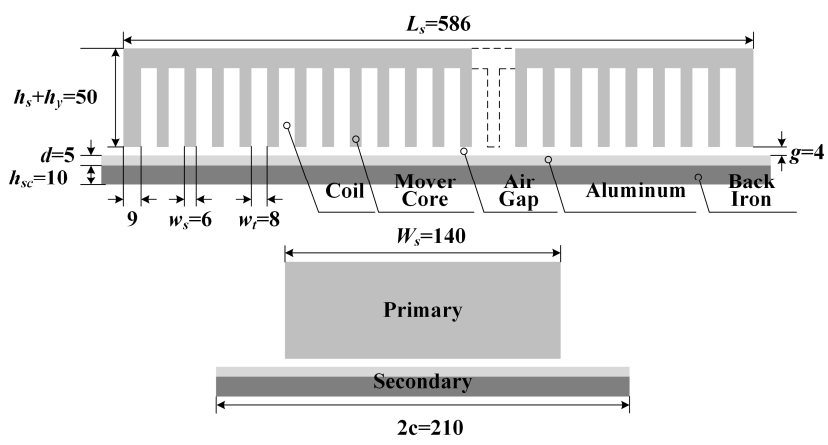

(b)

Fig. 1. (a) Manufactured model and (b) schematic diagram of LIMs



- $R_{l}$ - Primary winding resistance

- $L_{l}$ - Primary winding leakage inductance

- $R_{m}$ - Resistance corresponding to magnetic losses

- $L_{m}$ - Magnetizing inductance

- $R_{2}-$ Secondary resistance referred to the primary

- $L_{2}-$ Secondary leakage inductance referred to the primary

Fig. 2. Equivalent circuit of LIM

\subsection{Analytical calculation of primary winding parameter}

Fig. 2 shows the per-phase equivalent circuit of an LIM. In this figure, $\mathrm{R} 1$ is the per-phase resistance of the primary winding, which is calculated as follows:

$$
R_{1}=\frac{2 N\left(W_{s}+l_{e c}\right)}{\sigma_{w} A_{w}}
$$

where $N$ is the per-phase number of turns of the primary winding, $W_{s}$ is the primary width, $l_{e c}$ is the end connection length, $\sigma_{w}$ is the conductivity of the conductor used in the

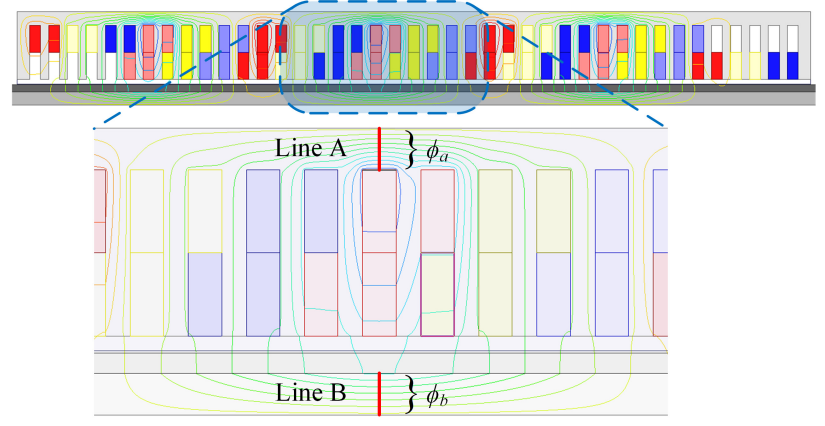

Fig. 3. FEA model for flux density analysis of LIM.

primary winding, and $A_{w}$ is the cross-sectional area of the conductor.

The primary leakage inductance is expressed as $[1,6,7]$

$$
L_{1}=\frac{2 \mu_{0} N^{2}}{p}\left[\frac{\left(\lambda_{s}+3 \lambda_{s} / 2 p+\lambda_{d}\right) W_{s}}{q}+\lambda_{e} l_{e c}\right]
$$

where $\mu_{0}$ is the permeability of vacuum, $p$ is the number of pole pairs, $q$ is the number of slots per pole per phase, $\lambda_{s}$ is the permeance of the slot, $\lambda_{d}$ is the end connection, and $\lambda_{e}$ is the differential [1]. The calculation results for $R_{l}$ and $L_{l}$ are 0.3 and $0.048 \mathrm{H}$, respectively.

\subsection{Leakage inductance analysis using FEA}

The leakage flux in LIMs refers to the flux that does not effectively act by being linked only to the primary conductor for flux action between the primary and secondary conductors [5]. The inductance is a proportional constant for the rate of change in flux and is expressed as follows:

$$
\lambda=L i=N \phi
$$

where $\lambda$ is the linkage inductance, $L$ is the winding inductance, $i$ is the input current, and $\varphi$ is the flux.

Therefore, the leakage inductance can be calculated by analyzing the leakage flux.

Fig. 3 shows an FEA model for analyzing the leakage flux. Fig. 4 shows the flux density in the primary and secondary cores. We denote the total flux that flows through the yoke of the primary core by $\varphi_{a}$. Similarly, we denote the flux that flows through the back iron of the secondary core over the airgap by $\varphi_{b}, \varphi_{a}-\varphi$ represents the leakage flux in the primary winding excitation [5]. After setting each line in the primary and secondary of the LIM, we calculate the flux density for the line using FEA. The flux density can be calculated using the flux by the following equation:

$$
\int_{s} B_{\text {LineA }}-B_{\text {LineB }} d s=\phi_{a}-\phi_{b}=\phi_{\text {Leak }}
$$



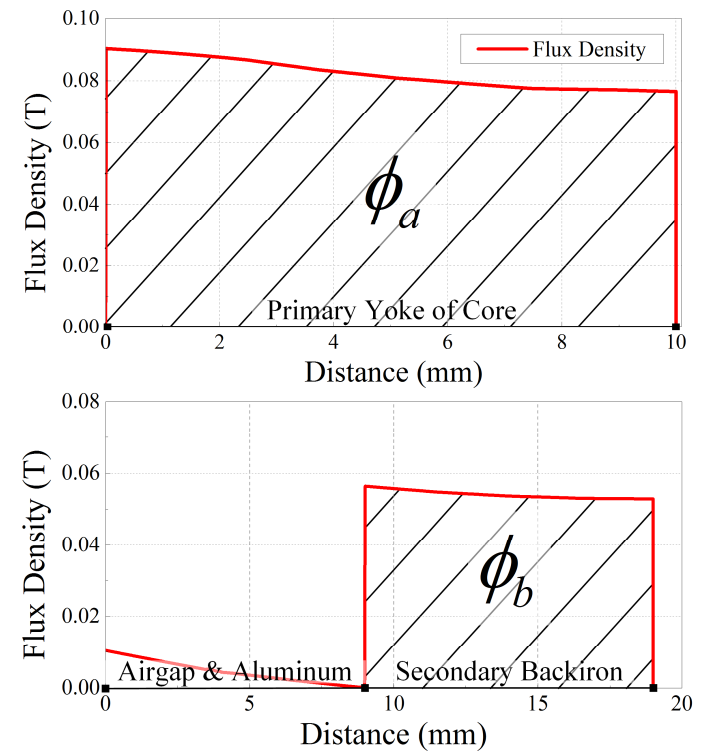

Fig. 4. Flux density in the primary and secondary cores

The leakage inductance calculated by the difference in the effective flux between the primary and secondary is $0.029 \mathrm{H}$. We derived the result of showing a difference of $0.019 \mathrm{H}$ from the leakage inductance value calculated by the preceding analytical equation.

\subsection{Leakage inductance calculation by experiment}

Similar to RIMs, the equivalent circuit parameters of LIMs can be calculated by the no-load and blocked-mover tests. In this section, we present the calculation of the equivalent circuit parameter by an experiment using a manufactured model, as shown in Fig. $1 . L_{1}+L_{m}$ and $R_{m}$ can be calculated by the no-load test as follows:

$$
\begin{gathered}
R_{m}=\frac{P_{N L} / 3-I_{N L}^{2} R_{1}}{I_{N L}^{2}} \\
L_{1}+L_{m}=\frac{\sqrt{\left(V_{N L} / I_{N L} \sqrt{3}\right)^{2}-\left(P_{N L} / 3 I_{N L}^{2}\right)^{2}}}{\omega}
\end{gathered}
$$

where $P_{N L}$ is the no-load input power, $I_{N L}$ is the no-load input phase current, $V_{N L}$ is the no-load input line voltage, and $\omega$ is the primary angular frequency.

Next, $L_{1}, L_{2}$, and $R_{2}$ can be calculated using the blockedmover test as follows (here, $L_{2}$ and $R_{2}$ are the equivalent circuit parameters of the rail, which is at the secondary side in the LIM):

$$
\begin{gathered}
R_{2}=\frac{P_{B L}}{3 I_{B L}^{2}}-R_{1} \\
L_{1}+L_{2}=\frac{\sqrt{\left(V_{B L} / I_{B L} \sqrt{3}\right)^{2}-\left(R_{1}+R_{2}\right)^{2}}}{\omega}
\end{gathered}
$$

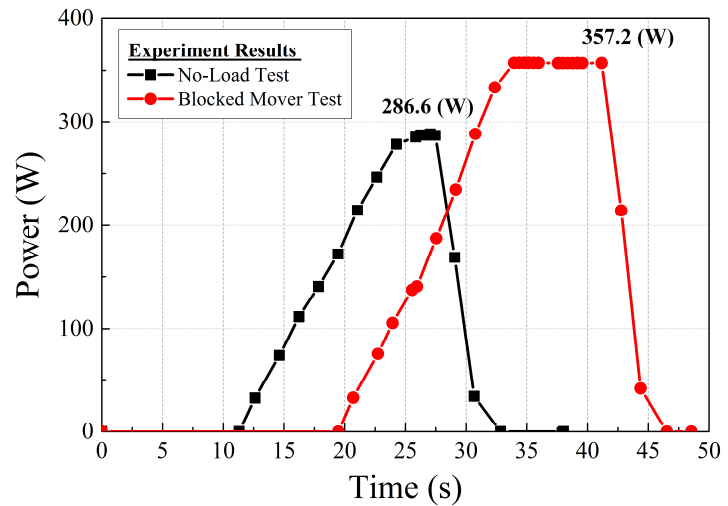

Fig. 5. Experiment results of the no-load and blocked-

\begin{tabular}{|c|c|c|c|c|}
\hline \multicolumn{5}{|c|}{ Experiment Results } \\
\hline \multicolumn{2}{|c|}{ PARAMETER } & \multicolumn{2}{|r|}{ No-Load Test } & Blocked Mover Test \\
\hline \multicolumn{2}{|c|}{ Input Power $(\mathrm{W})$} & \multicolumn{2}{|r|}{286.6} & 357.2 \\
\hline \multicolumn{2}{|c|}{ Input Voltage $\left(\mathrm{V}_{\mathrm{rms}}\right)$} & \multicolumn{2}{|r|}{45.6} & 45.5 \\
\hline \multicolumn{2}{|c|}{ Input Current $\left(\mathrm{A}_{\mathrm{rms}}\right)$} & & 3.7 & 4.1 \\
\hline \multicolumn{5}{|c|}{ Equivalent Parameter calculation } \\
\hline$R_{1}$ & $3.01(\Omega)$ & \multirow{3}{*}{$L_{1}$} & Analytical & $0.0483(\mathrm{H})$ \\
\hline$R_{m}$ & $4.3(\Omega)$ & & FEA & $0.0293(\mathrm{H})$ \\
\hline$L_{m}$ & $0.041(\mathrm{H})$ & & Experiment & $0.0286(\mathrm{H})$ \\
\hline
\end{tabular}
mover test.

Table 1. Experiment and Leakage inductance calculation results

where $P_{B L}$ is the input power of the blocked-mover test, $I_{B L}$ is the input phase current, and $V_{B L}$ is the input line voltage.

Fig. 5 shows a graph of the input power experiment results for the no-load and blocked-mover tests. The specific experiment and calculation results are listed in Table 1.

According to the calculation results of the leakage inductance, the value obtained using FEA is closer to the value of the actual model calculated from the experiment than that obtained by Eq. (2). The edge effect is considered in the analytical equation, but the nonlinear characteristics are not. However, both are considered in 2D FEA; therefore, this difference can possibly be considered as the main reason of the different calculation results. To verify the accuracy of the leakage inductance calculation, we compared the voltage and current waveforms by conducting a blocked-mover test using FEA. Both the experiment and FEA restricted the primary mover, and a $65-\mathrm{V}$ phase voltage was then applied as the input power source. When only the voltage is applied in the experiment, the magnitude of the input current is automatically determined by the resistance and inductance, which are secondary reaction effects. For FEA of the LIM, $R_{l}$ and $L_{l}$, which are the primary circuit parameters, should be entered with the voltage. For $R_{l}$, we entered 3.01 $\Omega$, [the result calculated by Eq. (1)], and for $L_{l}$, we entered $0.0293 \mathrm{H}$ calculated by FEA, which shows that the FEA result is close to that of the experiment. Fig. 6 shows a graph 


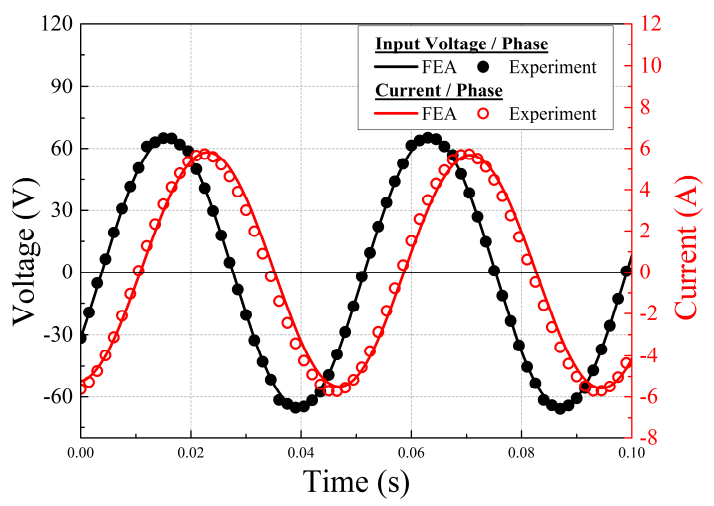

Fig. 6. Comparison of the input voltage and current results by experiment and FEA

comparing the voltage and current waveforms obtained from the experiment and by FEA. Because voltage was used as the input, the results of the experiment and FEA are the same. The current waveform may have a slightly different phase, but the magnitude is exactly the same. Therefore, we can conclude that the validity of the leakage inductance value calculated using FEA is very high.

\section{Characteristic Analysis of LIMs}

\subsection{Thrust characteristic}

In this section, we present the comparison of the thrust characteristics of the LIM as a function of the magnitude of the leakage inductance by deriving them using FEA. The thrust characteristics were analyzed by applying the leakage inductance calculated using the analytical equation and FEA. Fig. 7 shows a graph comparing the thrust characteristics as a function of the slip, and Fig. 8 shows a graph comparing the thrust characteristics as a function of speed. The difference in the magnitude of the thrust as a function of the difference in the input leakage inductance can thus be found. The difference in the magnitude of the leakage inductance is $0.019 \mathrm{H}$, which is a difference of approximately 1.6 times. The thrust is $2325 \mathrm{~N}$, which is also a difference of approximately 1.36 times the rated speed. Thus, if the leakage inductance is not accurately calculated in the thrust characteristic analysis of the LIM, the results can become greatly different, and the reliability of the analysis significantly declines.

\subsection{Machine capability curve}

To derive the equipment capability curve of the LIM, we present the V/f and variable frequency analyses by FEA. Fig. 9 shows the results of the V/F analysis. A constant thrust of $86.5 \mathrm{~N}$ can be generated at a rated speed of 1.6 $\mathrm{m} / \mathrm{s}$. Fig. 10 shows the FEA results for a variable frequency. A specific area can be identified by analyzing the thrust as

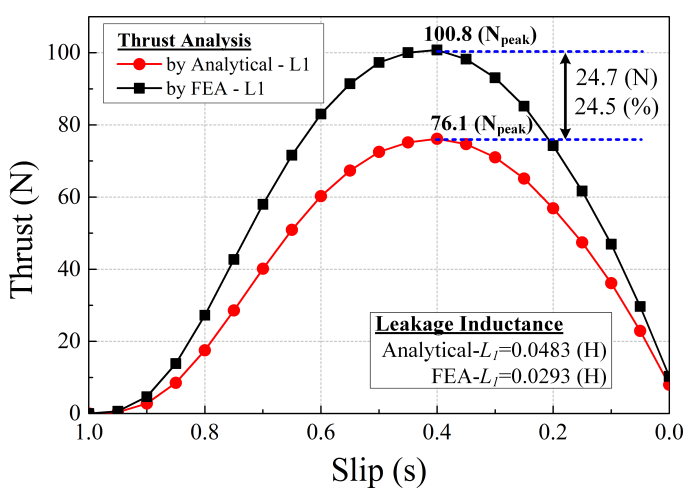

Fig. 7. Comparison of thrust analysis results according to slip



Fig. 8. Comparison of the thrust analysis results based on moving speed

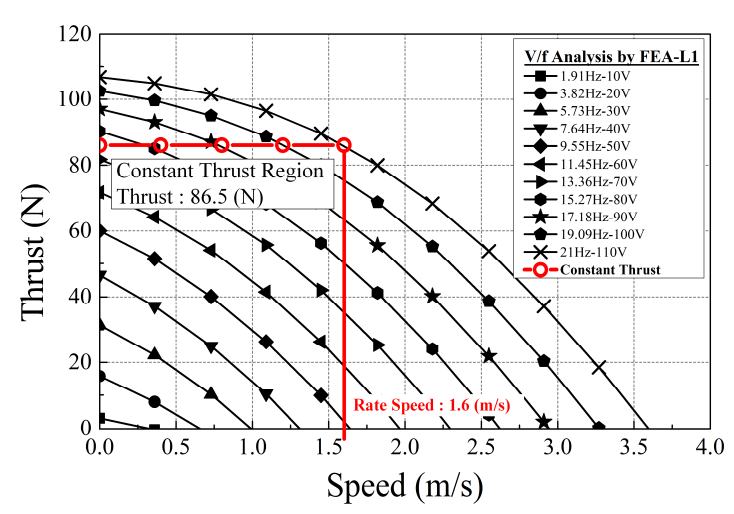

Fig. 9. Comparison of the thrust analysis results based on the slip

a function of the variable frequency in the rated operating condition. In addition, Fig. 10 shows the capability curve by applying the leakage inductance calculated by the analytical equation. We can possibly misinterpret the performance of the equipment because the overall thrust magnitude is very large. Therefore, we can conclude that the exact calculation of the leakage inductance is very important for the correct analysis and performance evaluation of LIMs. 


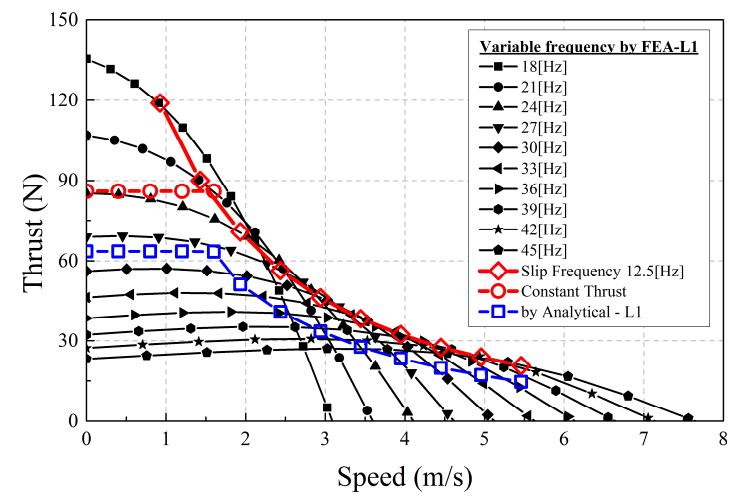

Fig. 10. Comparison of the thrust analysis results based on moving speed

\section{Conclusion}

In this paper, we have presented a means of accurately calculating the leakage inductance of LIMs, and we have analyzed and presented the characteristics as a function of the differences in the leakage inductance. The calculation of the leakage inductance was found to be highly valid because FEA derived a value closer to that of the actual production model than to that of the analytical equation. We derived the thrust characteristics and equipment capability curves by applying the leakage inductance calculated by the analytical equation and FEA to the voltage source analysis in FEA. The thrust characteristics caused by the difference in the leakage inductance were presented, and their influence was determined. We also found that the calculation of the leakage inductance is important for the exact design and analysis because it significantly affects the overall LIM capacity.

\section{Acknowledgements}

This research was a part of the project titled Research on fundamental core technology for ubiquitous whipping and logistics funded by the Ministry of Oceans and Fisheries, Korea.

\section{References}

[1] I. Boldea, "Linear Electric Motors, Englewood Cliffs," NJ, USA : Prectice-Hall, 2001.

[2] I. Boldea, "Linear Electric Machines, Drives, and MAGLEVs Handbook," CRC Press, Taylor and Francis Group, 2013.

[3] T. Nakata, and N. Takahashi, "Direct Finite Element Analysis of Flux and Current Distributions under Specified Conditions," IEEE Trans. Magn., vol. Mag 18, No. 2, pp. 325 330, March 1982.
[4] T. Nakata, N. Takahashi, and K. Fujiwara, "Efficient Solving Techniques of Martix Equations for Finite Element Analysis of Eddy Currents," IEEE Trans. Magn., vol. 24, No. 1, pp. 170 173, January 1988.

[5] Gubae Kang, Junha Kim, and Kwanghee Nam, "Parameter Estimation Scheme for Low-Speed Linear Induction Motors Having Different Leakage Inductances," IEEE Trans. Ind. Elect., vol. 50, no. 4, pp. 708-716, Aug. 2003.

[6] A. H. Isfahani, B. M. Ebrahimi, and H. Lesani, "Design Optimization of a Low-Speed Single-Sided Linear Induction Motor for Improved Efficiency and Power Factor," IEEE Trans. Magn., vol. 33, no. 2, pp. 266-272, Feb. 2008.

[7] A. Shiri, and A. Ahoulaie, "Design Optimization and Analysis of Single-Sided Linear Induction Motor, Considering All Phenomena," IEEE Trans. Energy Convers., vol. 27, no. 2, pp. 516-525, Jun. 2012.

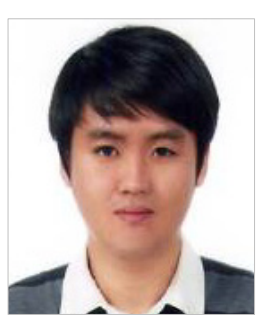

Jae-Hoon Jeong He received the B.S. degree in electric, electronic, and communication engineering education and M.S degree in electrical engineering from Chungnam National University, Daejeon, Korea, in 2011 and 2013 respectively. He is currently working for the Ph.D. degree in electrical engineering from Chungnam National University. His current research interests include design and analysis of motor/generator.



Jang-Young Choi $\mathrm{He}$ received his B.S., M.S., and Ph.D. degrees from Chungnam National University in 2003, 2005, and 2009, respectively. In 2009, he worked as a researcher in Halla Climate Control Corporation. August 2009, Dr. Choi joined as an assistant professor the Department of Electrical Engineering at Chungnam National University, Daejeon, Korea. His interests are in the design, analysis, simulation, and implementation of linear and rotary motors and permanent magnet generators.

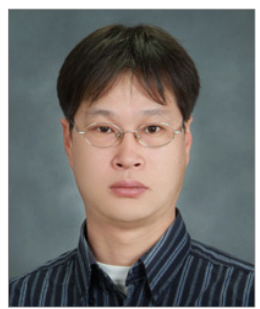

So-Young Sung He received the Ph.D. degree in electronic engineering from Chungnam National University, Korea, in 2013. Since 2009, he has been a senior researcher in KRISO (Korea Research Institute of Ships \& Ocean Engineering). His research interest is underwater acoustic network, navigation 
system for the digital ship, container X-ray scanning system, radiation detector for container vehicles, and motor control dynamic systems.

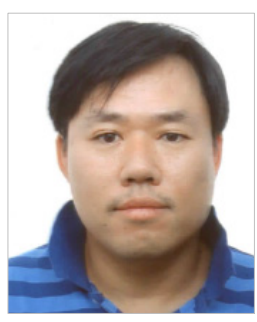

Jong-Won Park He received the Ph.D. degree in electronic engineering from A-Jou University, Suwon, Korea, in 2006. Since 1997, he has been a principal researcher in KRISO (Korea Research Institute of Ships \& Ocean Engineering). His research interest is underwater acoustic network, navigation system for the digital ship, container X-ray scanning system, radiation detector for container vehicles, and naval system technology.

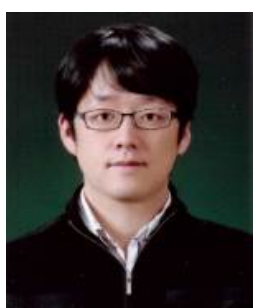

Jaewon Lim He received the B.S., M.S., and Ph.D. degrees in electrical and computer engineering from Seoul National University in 2004, 2006 and 2011 , respectively. Since 2011, he has been a senior researcher in KIMM (Korea Institute of Machinery and Materials). His research interest is the design of magnetic actuator and linear propulsion systems. 